\title{
Wie das Arzneimittelkompendium an den Nagel gehängt wird ...
}

\section{Cordula Boose}

Dr. med., Vizepräsidentin «Kunstausstellung der Schweizer Ärzte»

... auch das ist eine Kunst. Anlässlich der Jahresausstellung der «Kunstausstellung der Schweizer Ärzte» vom 12.7.-2.8.2015 im historischen Rathauskeller Murten führte Kinderärztin Antoinette Otz-Marbet vor, wie aus dem kiloschweren Arzneimittelkompendium ein federleichtes Kunstwerk werden kann. Viele Seiten wurden in Streifen geschnitten, gewässert, gezwirbelt, getrocknet, gefärbt, wieder getrocknet, erneut gezwirbelt, auf einem echten alten Spinnrad zu einem Papierfaden unterschiedlicher Dicke gesponnen und schliesslich zu dem Kunstwerk geformt, das in Murten zu sehen war.

Professor Dr. med. Rolf Zurbrügg, ehemaliger Chef der Kinderklinik Wildermeth in Biel, hielt anlässlich der Vernissage eine kleine Rede zum Thema «Arzt und Kunst», stellte die Parallelen zwischen ärztlicher Tätig-
Depuis 2015, le Salon suisse des médecins n'admet comme membre non seulement des médecins, leurs partenaires et des étudiants de médecine, mais aussi des personnes travaillant dans une branche médicale (psychologues, pharmaciens, dentistes, physiothérapeutes, etc.). Elles peuvent prendre part comme membre admis, avec les mêmes droits comme les membres actifs (exposition, vote à l'assemblée générale).

Nous aimerions bien gagner des nouveaux membres actifs aussi de la Romandie et du Tessin! Vous trouverez tous les détails sur notre homepage sous www.aerzte-kunst.ch ou vous pouvez vous informer chez notre présidente (Cordula Boose: corboose[at]aol.com).

Dal 2015 la «Kunstausstellung der Schweizer Ärzte» accoglie non solo medici di varie discipline, i loro partner attivi artisticamente e gli studenti di medicina, ma anche altri professionisti del settore medico (ad es. psicologi, assistenti di studio medico, arteterapeuti, ecc.). Questi ultimi, come membri ospiti, hanno gli stessi diritti dei membri attivi, vale a dire la possibilità di esporre e il diritto di voto all'assemblea generale.

Vorremmo che il numero dei membri attivi aumentasse, specialmente della Svizzera romanda e del Ticino, e invitiamo tutti i medici e i professionisti del settore che sono attivi artisticamente a informarsi sul nostro sito, www.aerzte-kunst.ch, e a diventare membri. Ulteriori informazioni possono essere ottenute anche presso l'autrice (Cordula Boose: corboose[at] aol.com).

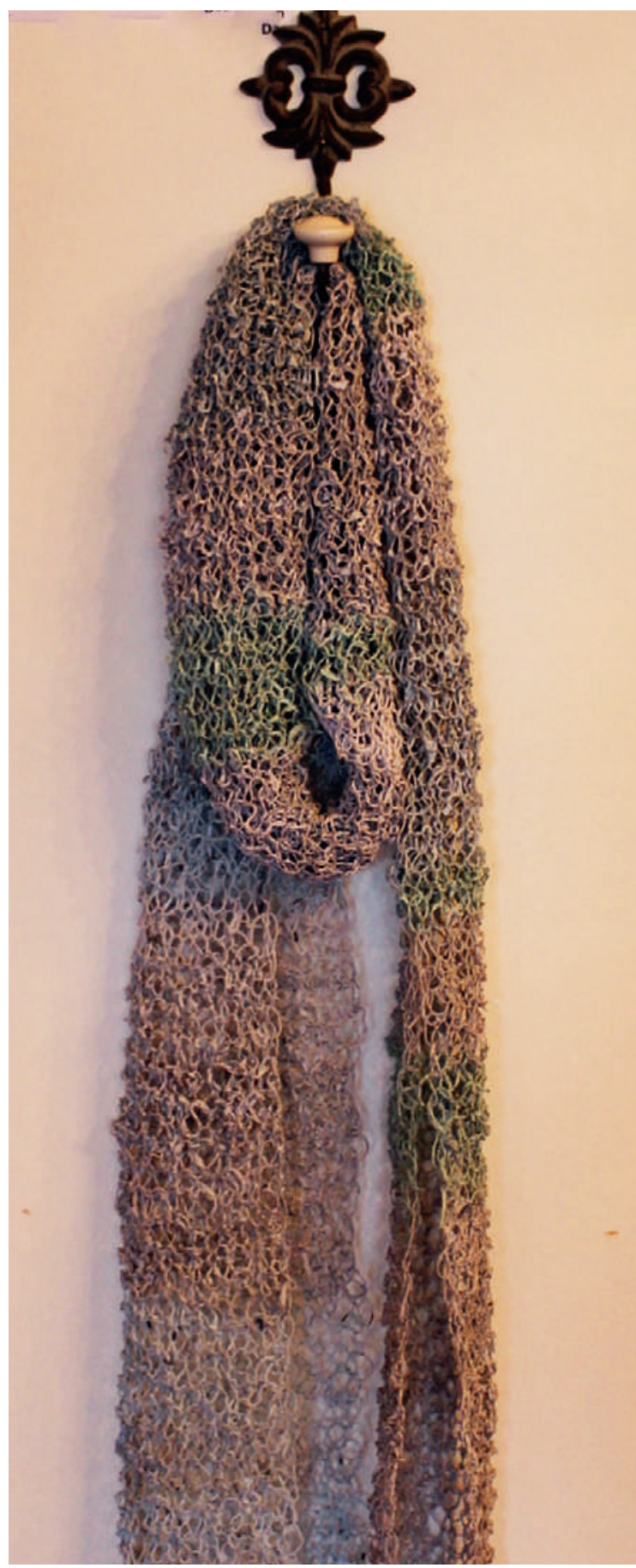

Das Arzneimittelkompendium an den Nagel gehängt (verstricktes Papier, 2012) - Antoinette Otz-Marbet 


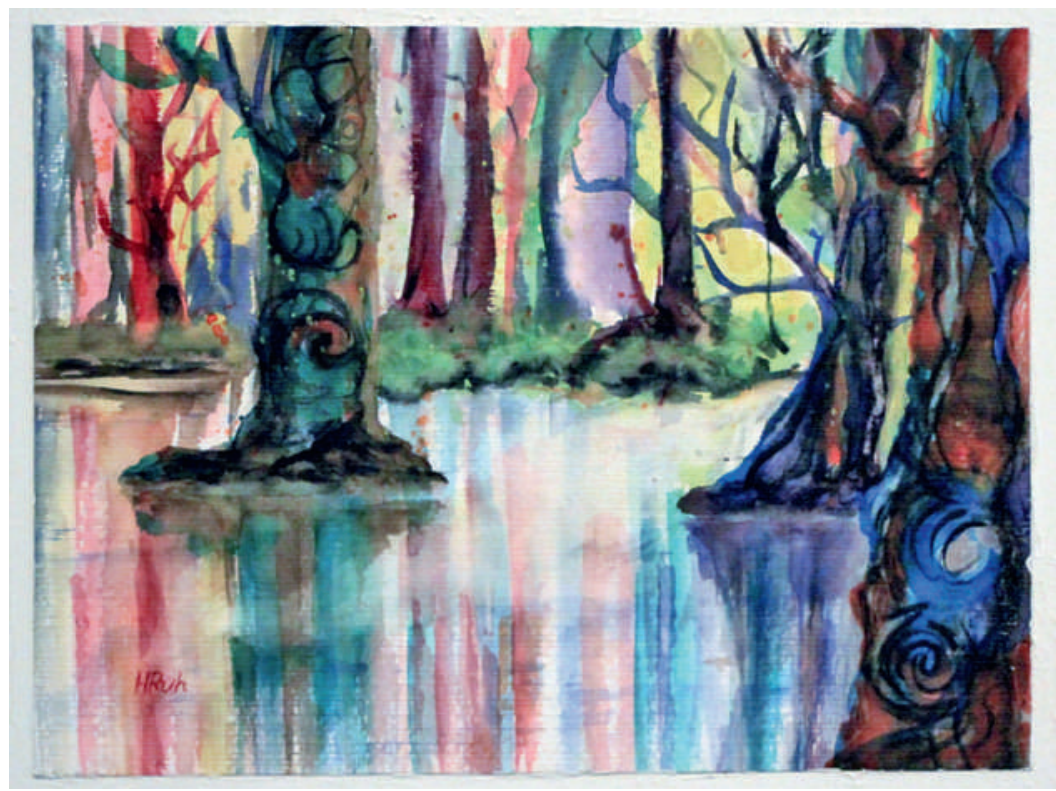

Spiegelung im Waldweiher (Aquarell, 2012) - Helene Ruh

keit und künstlerischem Schaffen dar und spannte so den Bogen von den anwesenden mehrheitlich ärztlichen Gästen zu den Besuchern, die einfach aus Freude am Schauen, an den unterschiedlichen Ausdrucksformen kreativer Betätigung den Weg aus der hochsommerlichen Hitze des Städtchens in den erfrischend kühlen Rathauskeller gefunden hatten. Im Anschluss an Herrn Prof. Zurbrügg ergriff Herr Stadtammann Christian Brechbühl spontan das Wort, begrüsste sehr freundlich die umfangreiche Gästeschar und brachte seine Freude über die neue Ausstellung zum Ausdruck. Der beschwingte "Ringelreihen» aus Weimutskiefer, die handschmeichlerisch rund geschliffene Skulptur "Ausblick» sowie die 4 Teilfiguren, die die Entstehung der endgültigen Form nachvollziehen lassen - die Holzskulpturen von Gallus Gadient eröffneten mit ihrer haptischen, olfaktorischen und visuellen Sinnlichkeit den Rundgang durch die vielgestaltige Ausstellung. Von fotografisch dokumentierten Japan-Impressionen von Agnes Berger Bertschinger führte der Weg vorbei an Blumenbildern in Acryl von Lis Weis über Helene Ruhs Aquarelle «Spiegelung im Waldweiher» und "Herbstwald mit Blick aufs Meer» zu WillyRené Felgenhauers Werken in Gouachetechnik, wie z.B. «Melano». Neben zarten Aquarellen mit argentinischen Stadt- und Hafenmotiven von Lili Cenoz hing als Kontrast Cordula Booses "La vida», ein Schiff, in Acryl gemalt auf eine alte Schranktür, mit einem Segel aus rostigem Blech. In kräftigen Farben stellte der kurz nach der Ausstellungseröffnung verstorbene Willy Bürgi noch seine «Farbsinfonien» vor. Lichtkugeln und kecke Ziegen aus Keramik in Rakutechnik von Monika
Wiesli brachten die handwerkliche Seite künstlerischer Tätigkeit zum Ausdruck, neben ausdrucksstarken Farbholzschnitten, wie z.B. "Orkney» von Peter Schlup und eher filigranen Holzschnitten («vor dem Spiegel») von Wolfgang Winkler. Roland Hofer stellte technisch sehr anspruchsvolle Collagen aus Fotos, Tusche, Acryl hinter Acrylglas vor; Roland Pickel zeigte seine Zeichnung "Thomas Hampson singt die Kindertotenlieder von Gustav Mahler».

Catherine Gobat schuf mit «Face nord» erneut ein grossformatiges «Spiegelbild» des Eigers. David Künzlers «Dach-Impressionen» neben dem «Urzustand» und dem "Andensturm» des leider ebenfalls im Herbst 2015 verstorbenen Rolf Weber, bunte abstrakte Bilder von Joanna Staeger neben Heinz Kellners grossformatigem und farbintensivem «night city». Da muteten die Blumen-Stillleben in Öl von Herwig Sausgruber fast schon im Stile alter Meister an. Eindrucksvolle Porträts, gemalt von Koni Bigler, Bernhard Wiesli («Frau von Srebrenica») sowie von Brigitte Fries («kämpfen sollst du», "Lebensreifeprüfung») vervollständigten die Ausdrucksvielfalt künstlerischen Schaffens von Ärzten und medizinisch Tätigen.

Kreativität, Ideen, Freude, Begeisterung für das, was man (er-)schafft, handwerkliche Arbeit, der richtige

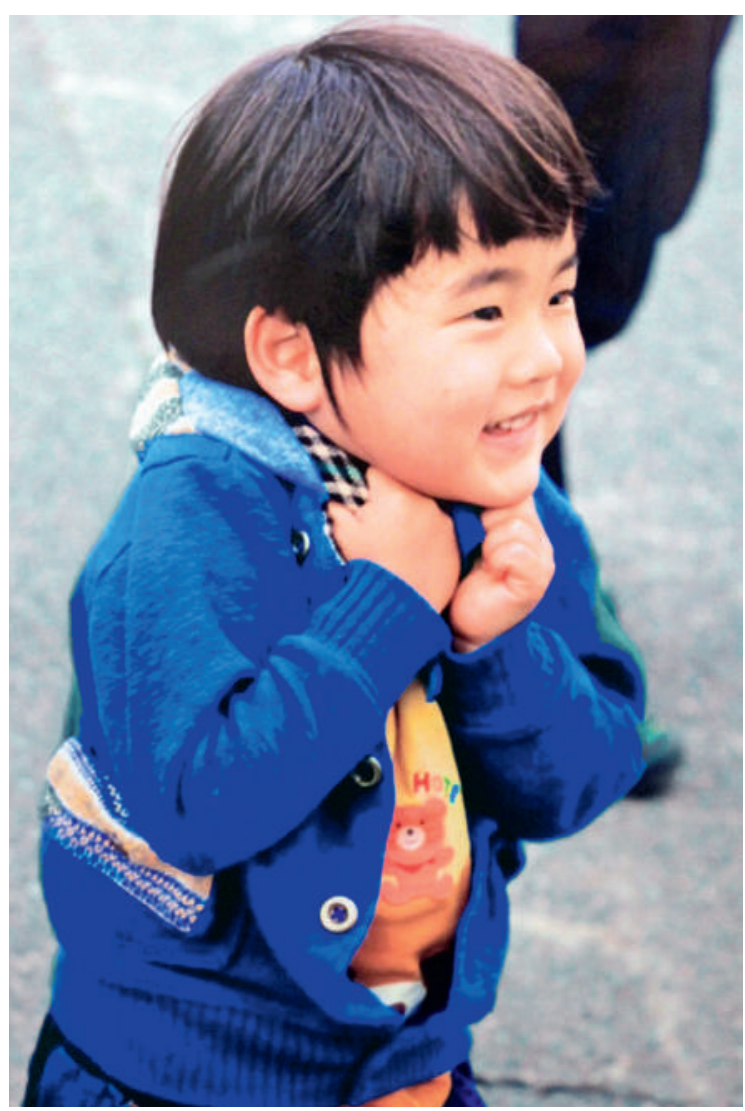

Haruyuki (Fotografie) - Agnes Berger Bertschinger 


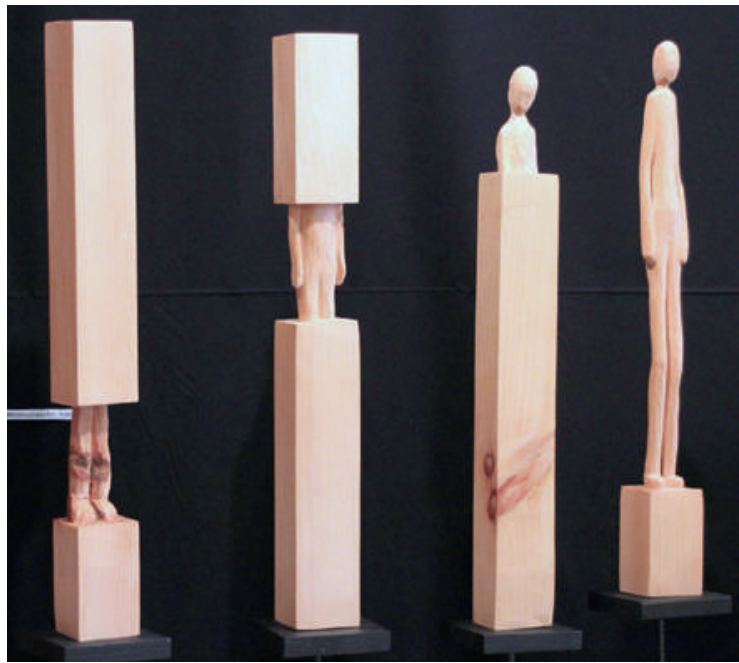

Einblicke (Weimutskiefer, natur, 2011) - Gallus Gadient

Blick und Bereitschaft zur dauernden Auseinandersetzung mit dem Werkstück sind wesentliche Bestandteile und Voraussetzungen für künstlerische Tätigkeit, unabhängig von Technik oder Motiv, in enger Parallele zur ärztlichen Tätigkeit. Vor allem aber bedeutet die künstlerische Betätigung Freude, Zufriedenheit, Stolz über Geschaffenes, Wagemut, etwas Neues entdecken, den Blick zu schärfen für Wesentliches, Konzentration auf Kleinigkeiten und dabei trotzdem nicht den Überblick zu verlieren, Freiraum für Gedanken und Gefühle, aber auch Selbstkritik, Selbstzweifel, Unvollendetes.

In die «Kunstausstellung der Schweizer Ärzte» werden seit 2015 nicht nur Ärzte und Ärztinnen verschiedener Fachrichtungen, deren kunstschaffende PartnerInnen sowie Studierende der Medizin aufgenommen, sondern es werden auch andere medizinisch tätige Personen, wie z.B. PsychologInnen, MPAs oder KunsttherapeutInnen, als Gastmitglieder aufgenommen mit den gleichen Rechten wie Aktivmitglieder, d.h., sie können ausstellen und in der Generalversammlung abstimmen.

Korrespondenz: Dr. med. Cordula Boose Vizepräsidentin «Kunstausstellung der Schweize Ärzte»

Ärztin für Psychiatrie und Psychotherapie Effingerstr. 92

CH-3008 Bern corboose[at]aol.com
Unsere nächste Jahresausstellung findet vom 16.7.6.8.2016 im historischen Rathaussaal in Murten statt und wird mit einer öffentlichen Vernissage am 16.7.2016 um 17 Uhr eröffnet.

Fotos:

Cordula Boose

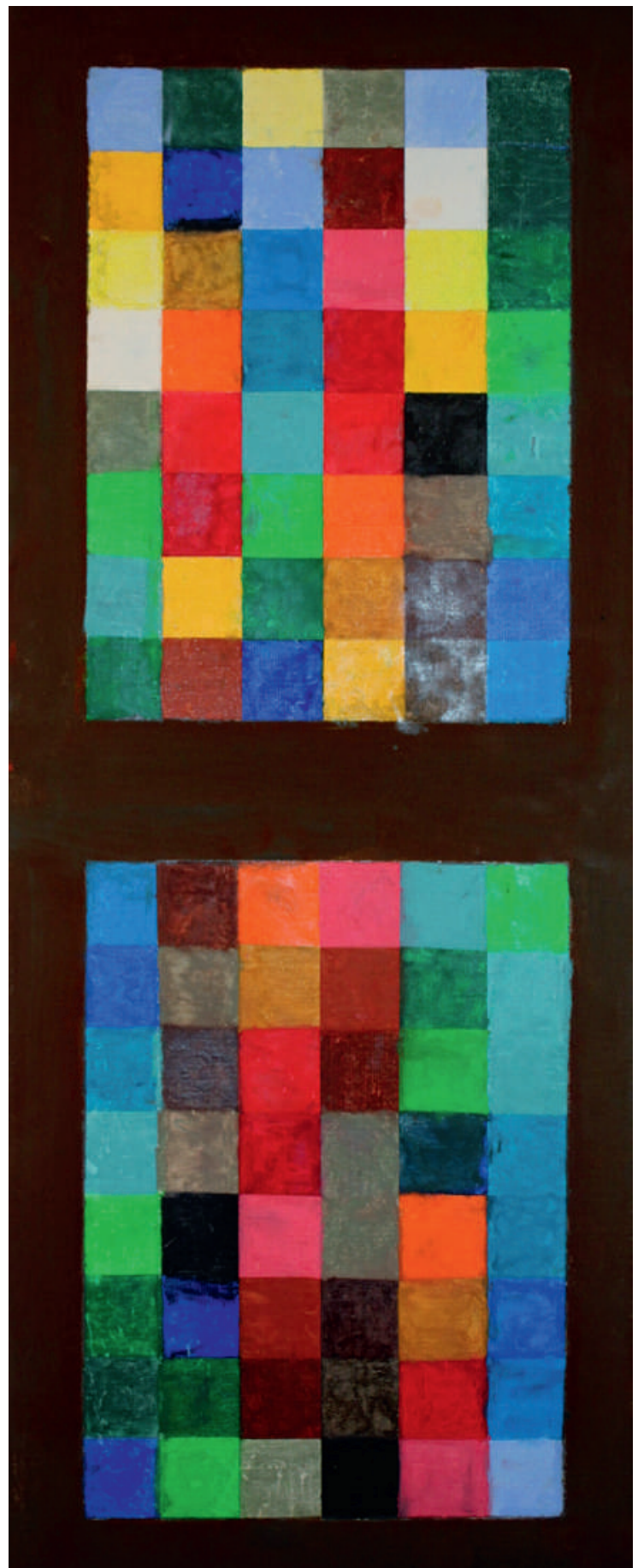

Farbsinfonie (Mischtechnik 2015) - Willy Bürgi

\section{Werden Sie Mitglied}

Wir möchten gern den Anteil von aktiven Mitgliedern, insbesondere aus der Romandie und aus dem italienischsprachigen Teil der Schweiz, erhöhen und laden daher alle interessierten künstlerisch tätigen Ärztlnnen und in der Medizin Tätigen ein, sich auf unserer Homepage www.aerzte-kunst.ch zu informieren und Mitglied zu werden. Nähere Auskünfte erhalten Sie auch gern bei der Autorin (corboose[at]aol.com). 